\title{
Multiphase Behavior of Tetraphenylethylene Derivatives with Different Polarities at High Pressures
}

\author{
Akihisa Miyagawa, $,{ }^{\dagger},{ }^{*}$ Tomokazu Kinoshita, ${ }^{\dagger}$ Yue Zheng, ${ }^{\S}$ Makoto Harada,${ }^{\dagger}$ \\ Gaku Fukuhara, ${ }^{\dagger, \|^{*}}$ and Tetsuo Okada ${ }^{\dagger *}$ \\ †Department of Chemistry, Tokyo Institute of Technology, Meguro-ku, Tokyo 152-8551, \\ Japan \\ ${ }^{\ddagger}$ Department of Chemistry, University of Tsukuba, Tsukuba, Ibaraki 305-8577, Japan \\ ${ }^{\S}$ Department of Chemistry, Beijing Key Laboratory for Microanalytical Methods and \\ Instrumentation, Key Laboratory of Bioorganic Phosphorus Chemistry and Chemical \\ Biology, Tsinghua University, Beijing 100084 (P. R. China) \\ "JST, PRESTO, 4-1-8 Honcho, Kawaguchi, Saitama 332-0012, Japan \\ Phone and Fax: +81-29-853-6914 \\ Email: miyagawa.akihisa.gf@u.tsukuba.ac.jp (A.M.) \\ Email: gaku@chem.titech.ac.jp (G.F.) \\ Email: tokada@chem.titech.ac.jp (T.O.)
}




\section{Abstract}

Although both pressure and temperature are essential parameters governing thermodynamics, the effects of the pressure on solution phase equilibria have not been well studied compared to those of temperature. Here, we demonstrate the interesting pressure-dependent behavior of tetraphenylethylene derivatives in multiphase systems composed of an organic phase and an aqueous phase in the presence and absence of $\gamma$ cyclodextrin $(\gamma-\mathrm{CD})$. In this system, tetraphenylethylene monocarboxylic acid (TPE1H) and its dicarboxylic acid $\left(\mathrm{TPE} 2 \mathrm{H}_{2}\right)$ are distributed in the aqueous phase and dissociated into the corresponding anions, that is, $\mathrm{TPE} 1^{-}$and $\mathrm{TPE} 2^{2-}$, when the $\mathrm{pH}$ is sufficiently high. The distribution ratios of TPE1H/TPE1 ${ }^{-}$and TPE2H/TPE2 ${ }^{2-}$ show opposing pressure dependencies: the distribution of the former in the organic phase increases with increasing pressure, whereas that of the latter decreases. The 1:1 complexation constants of TPE1' and TPE2 ${ }^{2-}$ with $\gamma-\mathrm{CD}$, which can be determined from the distribution ratios in the presence of $\gamma-C D$, also show opposing pressure dependencies: the former shows a positive pressure dependence, but the latter exhibits a negative one. These pressure effects on the distribution and complexation of TPE derivatives can be interpreted based on the differences in the molecular polarity of these solutes. The water permittivity is enhanced at high pressure, thus stabilizing the more polar TPE2 ${ }^{2-}$ in the aqueous phase to a larger 
extent than $\mathrm{TPE}^{-}$and, as a result, reduces its distribution in the organic phase, as well as its complexation with $\gamma$-CD. Fluorescence spectra in the aqueous phase suggest that the TPE derivatives form aggregates with $\gamma$-CD molecules, as detected by specific fluorescence. In addition, the fluorescence intensities of the $\gamma$-CD complexes are enhanced at high pressures because of the restricted rotation of the phenyl rings in the TPE molecules. This study provides new perspectives for multiphase partitioning and an attractive alternative to conventional extraction methods. 


\section{Introduction}

Chemical reactions and processes are mostly performed in a single phase. However, multiphase systems allow versatile reaction design and also act as a useful model for environmental and biological systems. For example, the two-phase partitioning of compounds an important chemical process in industry and the laboratory can be used widely in solvent extraction, ${ }^{1-5}$ solid phase extraction,,${ }^{6,7}$ as well as gas and liquid chromatography. ${ }^{8-11}$ The mass transfer of substances through biological membranes, ${ }^{12-16}$ drug deposition in organisms, ${ }^{17-19}$ and the dissolution of compounds of environmental importance ${ }^{20,21}$ can also be understood based on the two-phase partitioning principle. In particular, the partition constants of organic compounds between water and 1-octanol are extensively used as a convenient measure for predicting the efficiency of pharmaceuticals, environmental circulation of organic compounds, and chromatographic retention of organic solutes. $^{22-24}$

Multiphase partitioning is thermodynamically characterized by a partition constant, which is defined as the ratio of solute concentrations (activities) in two phases and can also be represented by the standard Gibbs energy of transfer of the solute between the two phases. ${ }^{25}$ Water (or an aqueous solution) is usually selected as a candidate of the two phases. Therefore, the other phase is chosen from a list of organic solvents immiscible 
with water considering solvent properties, such as polarity and proton donor and acceptor abilities In general, the appropriate organic solvent would be chosen from a limited scope, as the relative permittivity of a water-immiscible solvent typically ranges from 1.9 for hexane to approximately 10 for 1,2-dichloroethane. The solvent properties also depend on the temperature and pressure $(P)$, but the range of temperatures for exploration is limited because of the evaporation of liquids. However, in contrast, we can study the effects of pressure over a wider range. The pressure dependence of the permittivity has been reported for various solvents such as water, ${ }^{26}$ organic solvents, ${ }^{27-30}$ and thallous halides. $^{31}$ We have previously reported that the fluorescence spectrum of a mechanochromic molecule shows a red-shift under high pressures because of the increase in the solvent polarity around the molecule. ${ }^{32}$ Floriano et al. calculated the relative permittivity of water to be 89 at $400 \mathrm{MPa}$ and greater than 78 at $0.1 \mathrm{MPa} .{ }^{26}$ Moreover, Hegar et al. experimentally demonstrated the pressure dependence of the permittivity of water and confirmed the positive pressure dependence. ${ }^{33}$ On the other hand, the change in the permittivity $(\mathrm{d} \varepsilon / \mathrm{d} P)$ of the organic solvent is very small; for example, $3.1 \times 10^{-4}$ $\mathrm{MPa}^{-1}$ for ethyl acetate ${ }^{29}$ and $5.5 \times 10^{-3} \mathrm{MPa}^{-1}$ for chloroform. ${ }^{30}$ Thus, the partitioning between water and an organic solvent at high pressures should be dominantly governed by the changes in the properties of water. 
Host-guest and supramolecular reactions can also be considered multiphase reactions in some cases because a guest molecule is removed from the main solvent to form different species. Some of these reactions are also influenced by pressure. Hara et al. studied the solvation dynamics of a solvatochromic probe in a micellar phase and found that the polarity of the micelles decreases at high pressures because of the suppression of water penetration into the solvated region of the micelle. ${ }^{34}$ Marx et al. calculated the pressure effects on the water structure and solvation of osmolytes using ab initio molecular dynamics. ${ }^{35}$ The number of water molecules in a hydrophobic solvation shell around trimethylamine- $N$-oxide increased from 17 to 21 from 0.1 to $1 \mathrm{MPa}$, respectively. Cyclodextrin (CD) complexation is also influenced by pressure. ${ }^{36-40}$ Sueishi et al. reported the pressure dependence of the complexation of phenolic compounds with $\beta$-CD derivatives. ${ }^{40}$ For example, the complexation constant of 2-naphthol with permethylated $\beta-\mathrm{CD}$ at $80 \mathrm{MPa}$ is 1.6 times larger than that at $0.1 \mathrm{MPa}$. Thus, the complexation of $\mathrm{CD}$ should be affected by high pressures.

In this work, we studied the multiphase behavior of tetraphenylethylene (TPE) derivatives at high pressures. As typical aggregation-induced emission (AIE) fluorophores, TPE derivatives have been sufficiently studied and widely applied for fluorescent imaging, analyzing and materials. ${ }^{41-43}$ TPE emits no fluorescence when dissolved in a good solvent 
as a single molecule. However, when TPE at aggregate state or trapped in a CD cavity, ${ }^{44}$ strong emission will occur because of suppressed nonradiative decay caused by the restriction of intramolecular rotation of the phenyl rings. The pressure-dependent behavior of two TPE derivatives with one and two carboxylic groups in molecules were studied in a multiphase system composed of an organic solvent, aqueous phase, and $\gamma-C D$ in the aqueous phase. UV-absorption spectrometry in the organic phase provided quantitative information on the $P$-dependent liquid/liquid partition and CD-complexation of the TPE derivatives. In addition, fluorescence spectroscopy revealed the effects of pressure on the photophysical properties of the probes in the aqueous phase.

\section{Methods}

Synthesis of tetraphenylethylene monocarboxylic acid and dicarboxylic acid TPE monocarboxylic acid (TPE1H) and dicarboxylic acid $\left(\mathrm{TPE} 2 \mathrm{H}_{2}\right)$ were synthesized as reported previously, and the ${ }^{1} \mathrm{H}$ NMR data showed satisfactory agreement between the literature values (see Figure S1 in the Supporting Information(SI)). ${ }^{45,46}$ The structures of TPE1H and TPE2H $\mathrm{H}_{2}$ are shown in Figure 1.

Materials

$\gamma-\mathrm{CD}$ and tris(hydroxymethyl)aminomethane were purchased from Tokyo 
Chemical Industry Co., Ltd. (Tokyo, Japan). All solvents (hexane, chloroform, and ethyl acetate) were purchased from FUJIFILM Wako Pure Chemical Co. (Osaka, Japan).

Spectroscopic measurements under hydrostatic pressure

UV-vis and fluorescence spectra were measured under a range of hydrostatic pressures (0.1-320 MPa) using a custom-built high-pressure apparatus (Teramecs Co., Kyoto, Japan), as shown in Figure S2. A sample solution was filled and sealed in a quartz cell (inner dimension: $3 \mathrm{~mm}$ width $\times 2 \mathrm{~mm}$ thickness $\times 7 \mathrm{~mm}$ height) fitted to a short Teflon tube. Hydrostatic pressures in the range of 0.1 to $320 \mathrm{MPa}$ were applied to the sample solution. The high-pressure apparatus was then attached to each spectrometer to measure either the UV-vis absorption spectra (JASCO, V-560) or fluorescence spectra (JASCO, FP-8500). A circular dichroism spectrometer (JASCO, J-720WI) was used for measurements at ambient pressure.

Organic solvents were selected so that the $P$-dependence of the solute partition could be clearly measured. Preliminary experiments indicated that 2:8 mixtures of chloroform and hexane is suitable for $\mathrm{TPE} 1 \mathrm{H}$, and ethyl acetate for $\mathrm{TPE} 2 \mathrm{H}_{2}$. The aqueous solution was $100 \mathrm{mM}$ Tris buffer $\left(\mathrm{pH} 9.0\right.$ for $\mathrm{TPE} 1 \mathrm{H}$ and $\mathrm{pH} 7.4$ for $\mathrm{TPE}_{2} \mathrm{H}_{2}$ with varying concentrations of $\gamma-\mathrm{CD}$. The concentration range of $\gamma-\mathrm{CD}$ and the $\mathrm{pH}$ were also 
determined so that the measurable pressure dependence of the solute partition could be confirmed. The organic and aqueous phases in a 1:1 volume ratio were shaken in a $10 \mathrm{~mL}$ centrifuge tube for $30 \mathrm{~min}$, and then the phases were separated by centrifugation at 4000 rpm for $10 \mathrm{~min}$. For the UV-vis spectrometric measurements of the organic phase, the organic phase was first introduced into the cell, and, then, the aqueous phase was placed on the organic phase because the incident light passed through the lower half of the cell, as shown by the yellow ellipse in Figure S2. The order of phase introduction into the cell was reversed when the fluorescence spectra were measured for the aqueous phase. The less dense phase remained in the denser phase by adjusting the order of the phase introduction, for example, the aqueous phase $\left(0.997 \mathrm{~g} \mathrm{~cm}^{-3}\right)$ on ethyl acetate $(0.902 \mathrm{~g}$ $\left.\mathrm{cm}^{-3}\right)$.

Molecular orbital calculations

Molecular orbital (MO) calculations of TPE1- and TPE2 $\mathrm{H}^{2-}$ were performed using the semi-empirical quantum chemical program MOPAC 2016. ${ }^{47}$ The structure and dipole moment of TPE1 ${ }^{-}$and TPE $2 \mathrm{H}^{2-}$ were optimized using the semi-empirical PM7 method.

\section{Results \& discussion}

Liquid/liquid partitioning of TPE1H/TPE1 ${ }^{-}$and TPE2 $\mathrm{H}_{2} / \mathrm{TPE}^{2-}{ }^{-}$at high pressures 
The acid dissociation constant $\left(\mathrm{p} K_{\mathrm{a}}\right.$ ) of TPE1H was determined to be 5.89 by $\mathrm{pH}$ titration in a 1:3 water and methanol mixture. To evaluate the effects of methanol, the $\mathrm{p} K_{\mathrm{a}}$ of benzoic acid was assessed; $\mathrm{p} K_{\mathrm{a}}=4.75$ and 5.95 in $1: 1$ and $1: 3$ water and methanol mixtures, respectively. In water, the $\mathrm{p} K_{\mathrm{a}}$ of benzoic acid is 4.2 , as confirmed by a number of researchers. ${ }^{48}$ Thus, the $\mathrm{p} K_{\mathrm{a}}$ appears to increase with increasing methanol concentration. We speculate that the $\mathrm{p} K_{\mathrm{a}}$ of TPE1H in water is lower than that determined in 1:3 water/methanol and should be similar to that of benzoic acid $\left(\mathrm{p} K_{\mathrm{a}} \approx 4.2\right)$ in water.

The $P$-dependence of the partition ratio $\left(D^{\mathrm{TPE} 1}\right)$ of TPEH1 was studied between the aqueous phase at $\mathrm{pH} 9.0$ and 2:8 chloroform/hexane phase. In the aqueous phase, TPE1H is completely deprotonated into TPE $1^{-}$. Thus, $D^{\mathrm{TPE} 1}$ is given by

$$
\begin{gathered}
D^{\mathrm{TPE} 1}=\frac{[\mathrm{TPE} 1 \mathrm{H}]_{0}}{\left[\text { TPE1 }^{-}\right]_{\mathrm{W}}}, \\
\mathrm{TPE}^{-}(\mathrm{W}) \rightleftharpoons \mathrm{TPE} 1 \mathrm{H}(\mathrm{O}),
\end{gathered}
$$

where $\mathrm{O}$ and $\mathrm{W}$ denote the organic and aqueous phases, respectively. Figure $2 \mathrm{~A}$ shows the pressure dependence of $\ln D^{\mathrm{TPE} 1}$ for the TPE1H/TPE1 ${ }^{-}$system for $P=0.1-320 \mathrm{MPa}$. Obviously, $D^{\mathrm{TPE} 1}$ increases with increasing $P ; D^{\mathrm{TPE} 1}=14$ at $P=320 \mathrm{MPa}$, whereas $D^{\mathrm{TPE} 1}$ $=4.4$ at $P=0.1 \mathrm{MPa}$. From the $\ln D_{1}-P$ relationship, $\Delta V_{1}{ }^{\circ}=V_{1}(\mathrm{O})-V_{1}(\mathrm{~W})$ was estimated to be $-8.6 \mathrm{~cm}^{3} \mathrm{~mol}^{-1}$.

The $P$-dependence of the partitioning ratio for TPE2 $\mathrm{H}_{2}\left(D^{\mathrm{TPE} 2}\right)$ was also evaluated. The 
$\mathrm{p} K_{\mathrm{a}}$ values for the first and second deprotonation of stilbenedicarboxylic acid have been reported to be 4.03 and 4.06 , respectively. ${ }^{49}$ Because $\mathrm{TPE} 2 \mathrm{H}_{2}$ has a similar structure to stilbenedicarboxylic acid, its deprotonation constants may be in a similar range. We measured the partition ratio in the aqueous phase at $\mathrm{pH} 7.4$, and ethyl acetate. TPE2 $\mathrm{H}_{2}$ should be deprotonated into TPE $2^{2-}$ in the aqueous phase. Thus, $D^{\mathrm{TPE} 2}$ is defined as $D^{\mathrm{TPE} 2}=\frac{\left[\mathrm{TPE} 2 \mathrm{H}_{2}\right]_{0}}{\left[\mathrm{TPE}^{2-}\right]_{\mathrm{W}}}$,

$\mathrm{TPE}^{2-}(\mathrm{W}) \rightleftharpoons \mathrm{TPE}^{2} \mathrm{H}_{2}(\mathrm{O})$.

Figure 2B shows the $P$-dependence of $\ln D^{\mathrm{TPE} 2}$. In contrast to the pressure effect on $D^{\mathrm{TPE} 1}$, $D^{\mathrm{TPE} 2}$ decreases with increasing $P ; D^{\mathrm{TPE} 2}=0.74$ at $P=320 \mathrm{MPa}$ but $D^{\mathrm{TPE} 2}=1.3$ at $P=$ 0.1 MPa. The $\ln D_{2}-P$ relationship provides $\Delta V_{2}^{\circ}=V_{2}(\mathrm{O})-V_{2}(\mathrm{~W})=4.4 \mathrm{~cm}^{3} \mathrm{~mol}^{-1}$. Interestingly, the introduction of one carboxyl group on a TPE1H molecule leads to an entirely different partitioning pressure dependence. The partial molar volumes of various compounds were determined in water based on density measurements. The dissociation of the carboxyl group of benzoic acid in water induces a decrease in $\Delta V^{\circ}$ by $-11 \mathrm{~cm}^{3} \mathrm{~mol}^{-}$ ${ }^{1.50,51}$ In contrast, the dissociation of one carboxyl group is involved in a change in $\Delta V^{\circ}$ for the TPE1H/TPE1 ${ }^{-}$system, whereas the dissociation of the two carboxyl groups contributes to that of the TPE2 $\mathrm{H}_{2} / \mathrm{TPE} 2^{2-}$ system. If the volumes in the organic phase for TPE1H and TPE2 $\mathrm{H}_{2}$ are almost identical, $\Delta V^{\circ}$ for phase transfer can be estimated to be 
$\Delta V(\mathrm{~W})=V_{2}(\mathrm{~W})-V_{1}(\mathrm{~W})=\Delta V_{1}^{\circ}-\Delta V_{2}^{\circ}=-13 \mathrm{~cm}^{3} \mathrm{~mol}^{-1}$. This value almost corresponds to $\Delta V^{\circ}$ for the dissociation of one mole of carboxylic groups. ${ }^{52}$

Multiphase equilibrium in the presence of $\gamma$-CD in the aqueous phase at high pressures The complexation constants of TPE $1^{-}$and $\mathrm{TPE} 2^{2-}$ with $\gamma$-CD in the aqueous phase $\left(K_{\mathrm{CD}}\right)$ were evaluated from the dependence of the solute concentration in the organic phase on the $\gamma$-CD concentration in the aqueous phase. Assuming 1:1 complexation of the solutes with $\gamma-\mathrm{CD}$ in the aqueous phase, the partition ratio of TPE1H or TPE2 $\mathrm{H}_{2}\left(D_{\mathrm{CD}}^{\mathrm{TPE}}\right)$ is given by

$$
D_{\mathrm{CD}}^{\mathrm{TPE}}=\frac{[\mathrm{TPE}]_{\mathrm{O}}}{\left[\mathrm{TPE}^{-}\right]_{\mathrm{W}}+\left[\mathrm{TPE}^{-\cdot} \cdot \mathrm{CD}\right]_{\mathrm{W}}},
$$

where TPE represents TPE1H or TPE2H ${ }_{2}, \mathrm{TPE}^{-}$denotes $\mathrm{TPE}^{-}$or $\mathrm{TPE}^{2-}$, and $\mathrm{TPE}^{-}$. $\mathrm{CD}$ is the inclusion complex. This equation is rearranged into the following equation with the equilibrium concentration of $\gamma-\mathrm{CD}\left([\mathrm{CD}]_{\mathrm{w}}\right)$ in the aqueous phase:

$$
\frac{1}{D_{\mathrm{CD}}^{\mathrm{TPE}}}=\frac{1}{D^{\mathrm{TPE}}}+\frac{K_{\mathrm{CD} 1}}{D^{\mathrm{TPE}}}[\mathrm{CD}]_{\mathrm{w}},
$$

where $K_{\mathrm{CD} 1}$ is the $1: 1$ complexation constant of $\gamma-\mathrm{CD}$ with $\mathrm{TPE} 1^{-}$or TPE2 ${ }^{2-}$.

Figure 3 shows the relationships between $[\mathrm{CD}]_{\mathrm{w}}$ and $1 / D_{\mathrm{CD}}^{\mathrm{TPE} 1}$ and $1 / D_{\mathrm{CD}}^{\mathrm{TPE} 2}$ at $0.1,160$, and $320 \mathrm{MPa}$. Obviously, $1 / D_{\mathrm{CD}}^{\mathrm{TPE}}$ is not proportional to $[\mathrm{CD}]_{\mathrm{w}}$ in all cases, and the slope becomes larger with increasing $[\mathrm{CD}]_{\mathrm{w}}$. Figure 3 suggests that a higher-order inclusion 
complex is likely to be involved in partition; for example, a 1:2 (TPE1 $\left.{ }^{-}: \gamma-\mathrm{CD}\right)$ complex may form. If such species are formed in the aqueous phase, Equation (4) can be written as a polynomial of $[\mathrm{CD}] \mathrm{w}$,

$$
\frac{1}{D_{\mathrm{CD}}^{\mathrm{TPE}}}=\frac{1}{D^{\mathrm{TPE}}}+\frac{K_{\mathrm{CD} 1}}{D^{\mathrm{TPE}}}[\mathrm{CD}]_{\mathrm{W}}+f\left([\mathrm{CD}]_{\mathrm{w}}^{n}\right), n \geq 2
$$

where $f\left([\mathrm{CD}]_{\mathrm{w}}{ }^{n}\right)$ consists of quadratic or higher-order terms of $[\mathrm{CD}]_{\mathrm{w}}$. When 1:2 complexation occurs, $f\left([\mathrm{CD}]_{\mathrm{w}}\right)=\frac{K_{\mathrm{CD} 1} K_{\mathrm{CD} 2}}{D^{\mathrm{TPE}}}[\mathrm{CD}]_{\mathrm{w}}^{2}$, where $K_{\mathrm{CD} 2}$ is the consecutive $1: 2$ complexation constant. The plots in Figure 3 were analyzed assuming 1:2 complexation as a possible case. The fittings to the experimental results shown in green curves in Figure 3 are not perfect, but provisional complexation constants are summarized in Table S1. Because $K_{\mathrm{CD} 2}$ is larger than $K_{\mathrm{CD} 1}$ in some cases, these values are not reliable. This anomalous result obviously arises from the inappropriate assumption of 1:2 complexation. The species involving multiple $\gamma$-CD molecules, which were not specified at this stage, should be present in the aqueous phase; otherwise, the $[C D]_{\mathrm{W}}$ dependence of $\frac{1}{D_{\mathrm{CD}}^{\mathrm{TPE}}}$ cannot be interpreted. The above analysis indicates that the plots in Figure 3 cannot be explained by the usual multiple complexation of $\gamma-\mathrm{CD}$. This will be discussed later using the fluorescence spectra in the aqueous phase.

$K_{\mathrm{CD} 1}$ can be determined from the limiting slope at $[\mathrm{CD}]_{\mathrm{W}} \rightarrow 0$ because $f\left([\mathrm{CD}]_{\mathrm{w}}{ }^{n}\right)$ approaches zero. 


$$
\left(\frac{\mathrm{d}\left(1 / D_{\mathrm{CD}}^{\mathrm{TPE}}\right)}{\mathrm{d}[\mathrm{CD}]_{\mathrm{w}}}\right)_{[\mathrm{CD}]_{\mathrm{w}}=0}=\frac{K_{\mathrm{CD} 1}}{D^{\mathrm{TPE}}}
$$

Figure 4 shows the change in $\ln K_{\mathrm{CD} 1}^{\mathrm{TPE} 1}$ and that in $\ln K_{\mathrm{CD} 1}^{\mathrm{TPE} 2}$ with $P$. Interestingly, the former increases but the latter decreases with increasing $P$. From the results, $\Delta V$ for the complexation of $\mathrm{TPE}^{-}$with $\gamma-\mathrm{CD}$ was determined to be $-6.1 \mathrm{~cm}^{3} \mathrm{~mol}^{-1}$, and the corresponding value for TPE2 $2^{2-}$ is $4.2 \mathrm{~cm}^{3} \mathrm{~mol}^{-1}$.

The configuration of the TPE molecules in the cavity of $\gamma-\mathrm{CD}$ was studied using spectroscopy and molecular simulation. Figure S3A shows the UV and circular dichroism spectra, as well as the anisotropy $(g)$ factor $(\Delta \varepsilon / \varepsilon)$ of the TPE1 ${ }^{-1} \gamma-\mathrm{CD}$ system. The configuration of TPE can be interpreted from the Cotton effect confirmed in the circular dichroism spectra, which provides information on the conformational and structural features of a molecule. ${ }^{53}$ A negative Cotton effect was observed at $286 \mathrm{~nm}$, which was induced by the complexation of TPE $1^{-}$with $\gamma$-CD. Similarly, a negative Cotton effect was observed at $287 \mathrm{~nm}$ for TPE2 $2^{2-} / \gamma-\mathrm{CD}$, as shown in Figure S3B. The $g$-factor values (0.002) of the negative peaks are relatively large compared to other cases, ${ }^{54}$ suggesting that both TPE1 ${ }^{-}$and TPE2 ${ }^{2-}$ penetrate the $\gamma$-CD cavity deeply. Figures S4A and S5A show the dipole moments of TPE1 ${ }^{-}$and TPE2 ${ }^{2-}$ calculated using MOPAC $2016 .{ }^{47}$ The dipole moment of TPE1 ${ }^{-}$lies in the direction perpendicular to the ethylene double bond. The molecular orientation of $\mathrm{TPE}^{-}$in the $\gamma$-CD cavity can be determined based on the sector 
rule. ${ }^{55,56}$ The negative Cotton effect indicates that the dipole moment lies in the direction of the negative region outside the sector cone, as shown in Figure S4B. Thus, the ethylene double bond is aligned parallel to the direction connecting the open cavities of the $\gamma-\mathrm{CD}$ molecule. X-ray crystallography measurements of the $\beta$-CD complex of benzoic acid have shown that the carboxyl group of benzoic acid points to the smaller rim of the CD cone. ${ }^{57}$ Wei et al. also reported a similar location of the carboxyl group of an ursolic acid molecule in a $\gamma$-CD complex. ${ }^{58}$ Thus, one carboxyl acid should be present on the narrower rim of the $\gamma-\mathrm{CD}$ cone. The dipole moment of TPE2 $2^{2-}$ lies in the direction perpendicular to the ethylene double bond (Figure S5A). Thus, TPE2 ${ }^{2-}$ is accommodated in the $\gamma$-CD cavity in a similar way to TPE1- Hence, the structures of the $\gamma$-CD complexes can be reasonably inferred, as shown in Figures S4B and S5B. These different structures should lead to different hydration structures of the complexes. This is reflected in the $\Delta V$ values for complexation.

Fluorescence spectra in the aqueous phase during multiphase partition Fluorescence spectra were measured in the aqueous phase to provide further insight into the pressure effect on the multiphase behavior of TPE. Figure 5 summarizes the effect of the pressure on the fluorescence spectra of $\mathrm{TPE} 1^{-}$and TPE2 $2^{2-}$ in the aqueous phases containing $\gamma$-CD equilibrated with the organic phases. The fluorescence intensities 
increase with increasing pressure for both $\mathrm{TPE} 1^{-}$and TPE $2^{2-}$. The three vibrational bands observed at 363, 379, and $404 \mathrm{~nm}$ are assigned to monomer fluorescence and the broad band at $420-530 \mathrm{~nm}$ is assigned to aggregate fluorescence. ${ }^{44}$ Although aggregate fluorescence is obvious at $0.1 \mathrm{MPa}$, that from the monomer becomes dominant with increasing pressure. Figure 5C and D show the normalized spectra, indicating that the relative contribution from the aggregate fluorescence decreases with increasing pressure. As discussed previously, the relationships between $1 / D_{\mathrm{CD}}^{\mathrm{TPE}}$ and $[\mathrm{CD}]_{\mathrm{w}}$ were nonlinear in all cases. Although this suggests the formation of species involving multiple CD molecules, 1:2 (TPE:CD) complexation was unlikely. We conclude that the TPE aggregates detected by fluorescence measurements consist of several TPE and $\gamma$-CD molecules and cause a nonlinear relationship between $1 / D_{\mathrm{CD}}^{\mathrm{TPE}}$ and $[\mathrm{CD}]_{\mathrm{w}}$. The partition ratios and complexation constants allow the estimation of the concentrations of TPE species in the aqueous phase. In this estimation, the formation of a 1:1 complex and aggregates was considered. Figure $5 \mathrm{~A}$ and $\mathrm{B}$ show the $P$-dependencies of the concentrations of the TPE $1^{-}$- and TPE $2^{2-}$-related species. Because the compositions of the aggregates are not known, the concentration of $\mathrm{TPE}^{-}$or $\mathrm{TPE}^{2-}$ contained in the aggregates is plotted in each figure. Details of the estimation of the concentrations are described in the SI. A marked contrast is seen in the $P$-dependence of the concentrations 
of free $\mathrm{TPE}^{-}$and $\mathrm{TPE} 2^{2-}$; the concentration of $\mathrm{TPE}^{-}{ }^{-}$decreased, whereas that of TPE2 ${ }^{2-}$ increased with increasing pressure. This reflects the different $P$-dependencies of $D^{\mathrm{TPE} 1}$ and $D^{\mathrm{TPE} 2}$. In addition, the TPE1- concentration in the aggregate is almost constant over the entire pressure range, whereas that of TPE $2^{2-}$ decreases with increasing pressure. This result is consistent with those from the fluorescence spectra, which indicate that the fluorescence from the aggregates does not show a clear $P$-dependence (Figure 6C andD). Interestingly, the concentrations of the $\gamma$-CD complexes of TPE $1^{-}$and TPE2 ${ }^{2-}$ are almost constant over the entire pressure range; this is due to the opposing $P$-dependencies of $D^{\mathrm{TPE}}$ and $K_{\mathrm{CD} 1}^{\mathrm{TPE}}$.

The vibrational bands mainly arise from the monomer TPE molecule accommodated in the $\gamma$-CD cavity, for which the rotation of the phenyl rings of the TPE molecule is strongly inhibited. Therefore, simply, the increase in fluorescence intensity with increasing $P$ suggests an increase in the concentration of the $\gamma-\mathrm{CD}$ complex. However, the complex concentration is almost independent of the pressure. Hence, these results strongly suggest that the rotation of phenyl rings in the TPE molecules is more effectively inhibited in the $\gamma$-CD complex at high pressures, leading to more intense fluorescence.

\section{Conclusion}


The pressure has not been regarded as an important factor in the multiphase partition of compounds. Here, we discuss the results obtained for the multiphase partition of TPE derivatives under hydrostatic pressures. TPE1H/TPE1 ${ }^{-}$and TPE2 $\mathrm{H}_{2} / \mathrm{TPE}^{2-}$ show different $P$-dependencies in two-phase partition and $\gamma$-CD complexation. These behavior can be interpreted by the permittivity of water. Because this paper presents the first liquidliquid partition study under hydrostatic pressures, the qualitative discussions based on the reported $P$-dependence of water permittivity are meaningful. As indicated earlier, the permittivity of water increases with increasing pressure. ${ }^{33}$ A hydrophobic molecule escapes from the aqueous phase into the organic phase or the cavity of $\gamma-\mathrm{CD}$ at high pressures, whereas the hydrophilic molecule remains in the aqueous phase. In the present instance, this mechanism leads to two contrasting findings; that is, the concentration of free TPE1 ${ }^{-}$in the aqueous phase decreases but that of TPE2 increases with increasing pressure.

Although the pressure is a critical experimental parameter that governs the partition of organic molecules, its use has been limited compared to that of other physical parameters such as temperature. If a large amount of data is compiled, multiphase partitioning can be conducted by changing the pressure rather than changing the organic solvent. In addition, the pressure effect on multiphase partition is related to the retention 
mechanism in liquid chromatography, the molecular behavior in biological cells, and geological phenomena occurring in the deep sea. ${ }^{59}$ We believe that the present concept will draw the attention of researchers in a range of fields, including analytical chemistry, as well as the environmental and life sciences.

\section{Supporting Information}

The Supporting Information is available free of charge on the ACS Publications website at DOI:

Estimation of the concentrations of TPE1H and TPE2 $\mathrm{H}_{2}$ species in the aqueous phase; Provisional $K_{\mathrm{CD} 1}$ and $K_{\mathrm{CD} 2}$ values at $0.1,160$, and $320 \mathrm{MPa}$; NMR spectra for TPE1H and TPE2 $\mathrm{H}_{2}$; Schematic illustration of the pressure apparatus and cell; UV spectra, CD spectra, and $g$ factor for the complexes of $\gamma$-CD and TPE1 and for the complexes of $\gamma$ CD and TPE2; Calculated dipole moment of TPE1 and configuration of the complex of $\gamma$-CD and TPE1; Calculated dipole moment of TPE2 and configuration of the complex of $\gamma$-CD and TPE2.

\section{Acknowledgement}

This work was supported by a Grant-in-Aid for Scientific Research (A) (No. 17H01209) and (B) (No. 19H02746) from JSPS, Japan Science and Technology Agency (JST), PRESTO (No. JPMJPR17PA), and the Re-Inventing Japan Project (Type A-1: CAMPUS Asia Program) funded by MEXT. The program name is Tokyo Tech CAMPUS Asia Summer School (Research-Oriented Program). 
(a)<smiles>O=C(O)c1ccc(C(=C(c2ccccc2)c2ccccc2)c2ccccc2)cc1</smiles>

(b)<smiles>O=C(O)c1ccc(C(=C(c2ccccc2)c2ccccc2)c2ccc(C(=O)O)cc2)cc1</smiles>

Figure 1 Structures of (a) TPE1H and (b) TPE2 $\mathrm{H}_{2}$. 
A

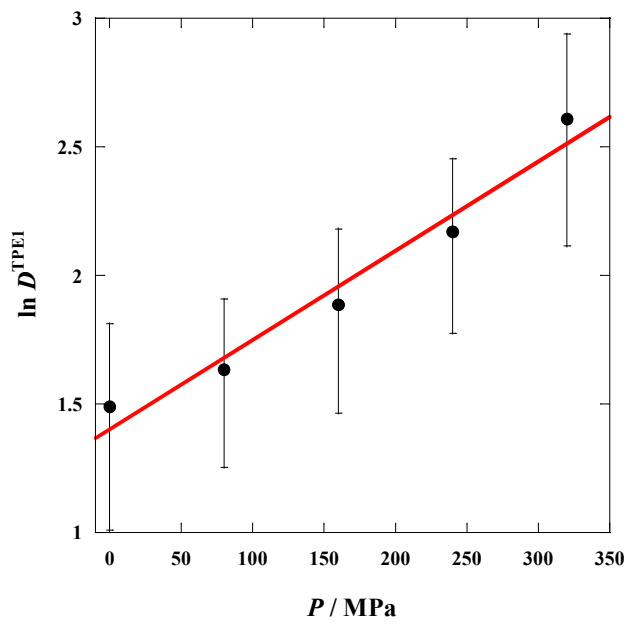

B

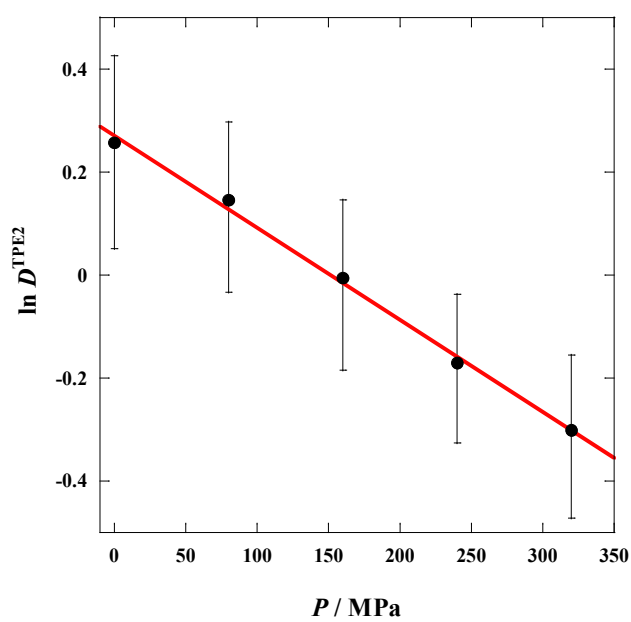

Figure $2 P$-dependencies of the partition ratios between the organic and aqueous phases in TPE1H/TPE1 ${ }^{-}$and TPE2 $\mathrm{H}_{2} / \mathrm{TPE}^{2-}$ systems. The correlation coefficients of TPE1H/TPE1 ${ }^{-}$and TPE2H $\mathrm{H}_{2} / \mathrm{TPE}^{2-}(r)$ are 0.982 and 0.998 , respectively. 
TPE1
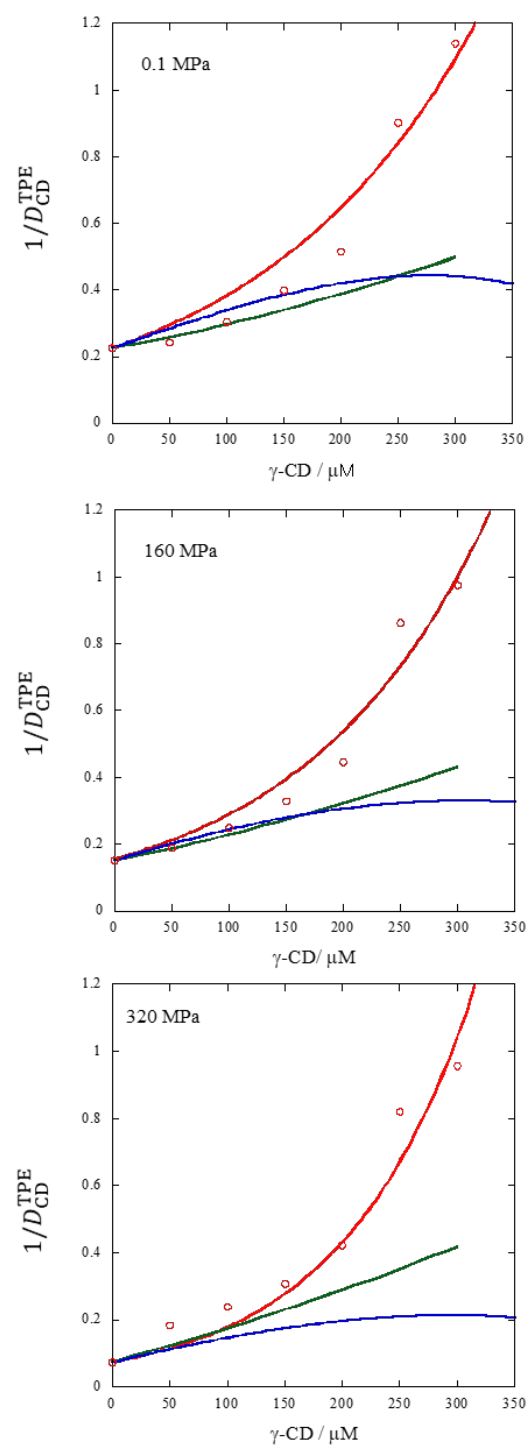

TPE2
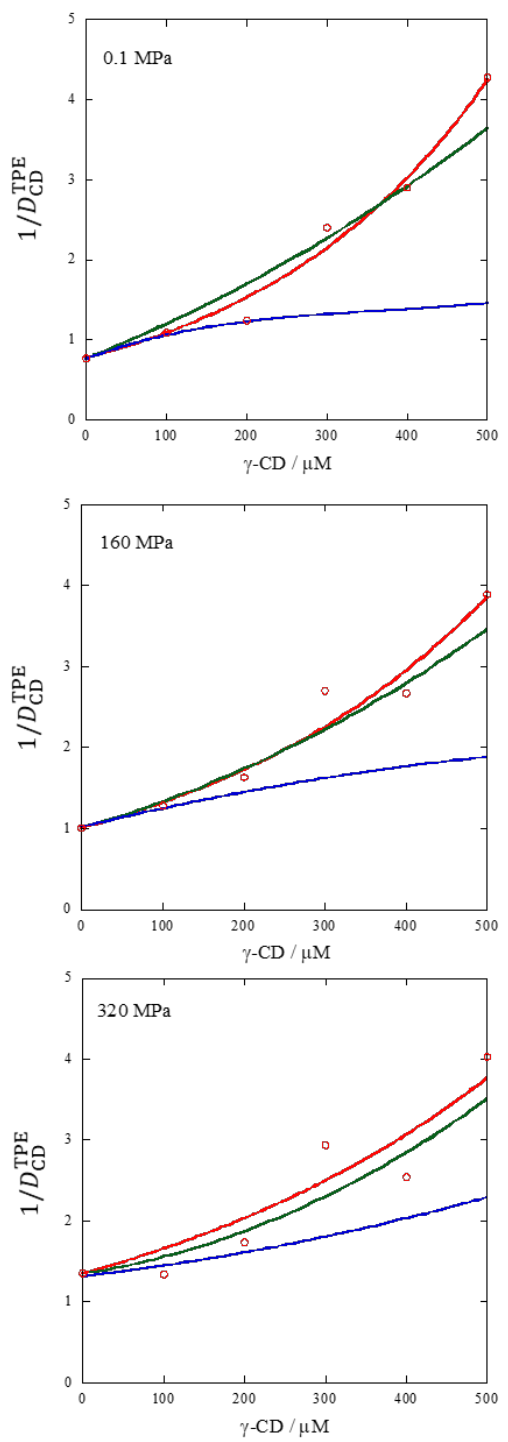

Figure 3 Dependences of $1 / D_{\mathrm{CD}}$ on the $\gamma-\mathrm{CD}$ concentration in the aqueous phase at 0.1 , 160 , and $320 \mathrm{MPa}$. Plots and experimental values. Red curves are eye guides. Blue curves plot data assuming that the 1:1 CD complex is the only aqueous-phase species containing $\gamma$-CD. Green curves assume the formation of 1:1 and 1:2 CD complexes in the aqueous phase. 


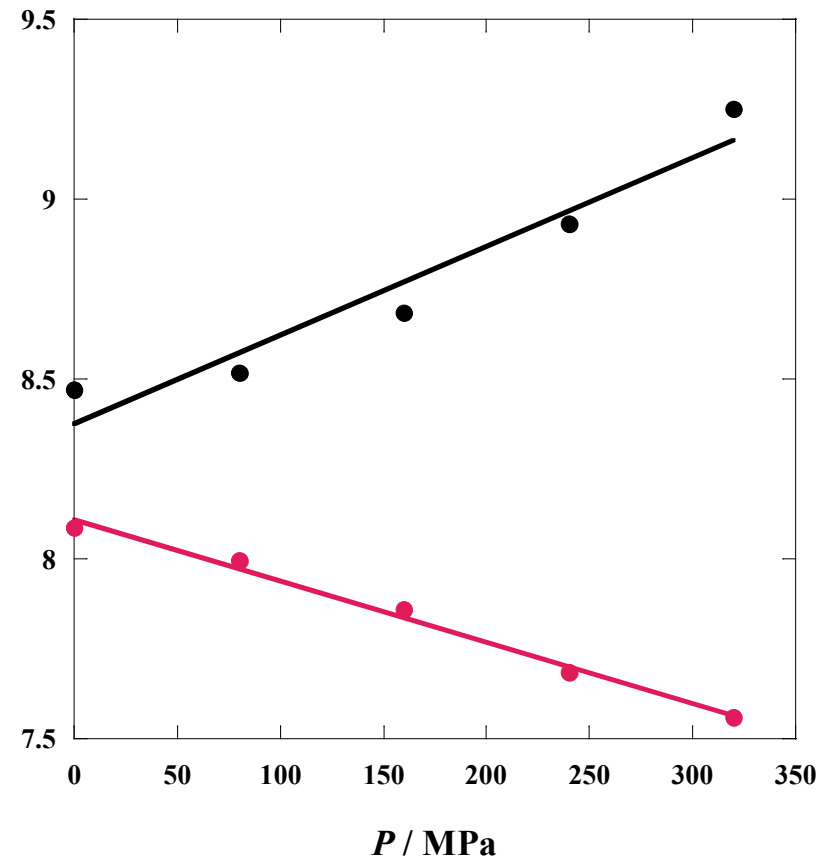

Figure $4 P$-dependence of $\ln K_{\mathrm{CD} 1}^{\mathrm{TPE} 1} \quad($ black, $r=0.966)$ and $\ln K_{\mathrm{CD} 1}^{\mathrm{TPE} 2} \quad($ red, $r=0.995)$. 

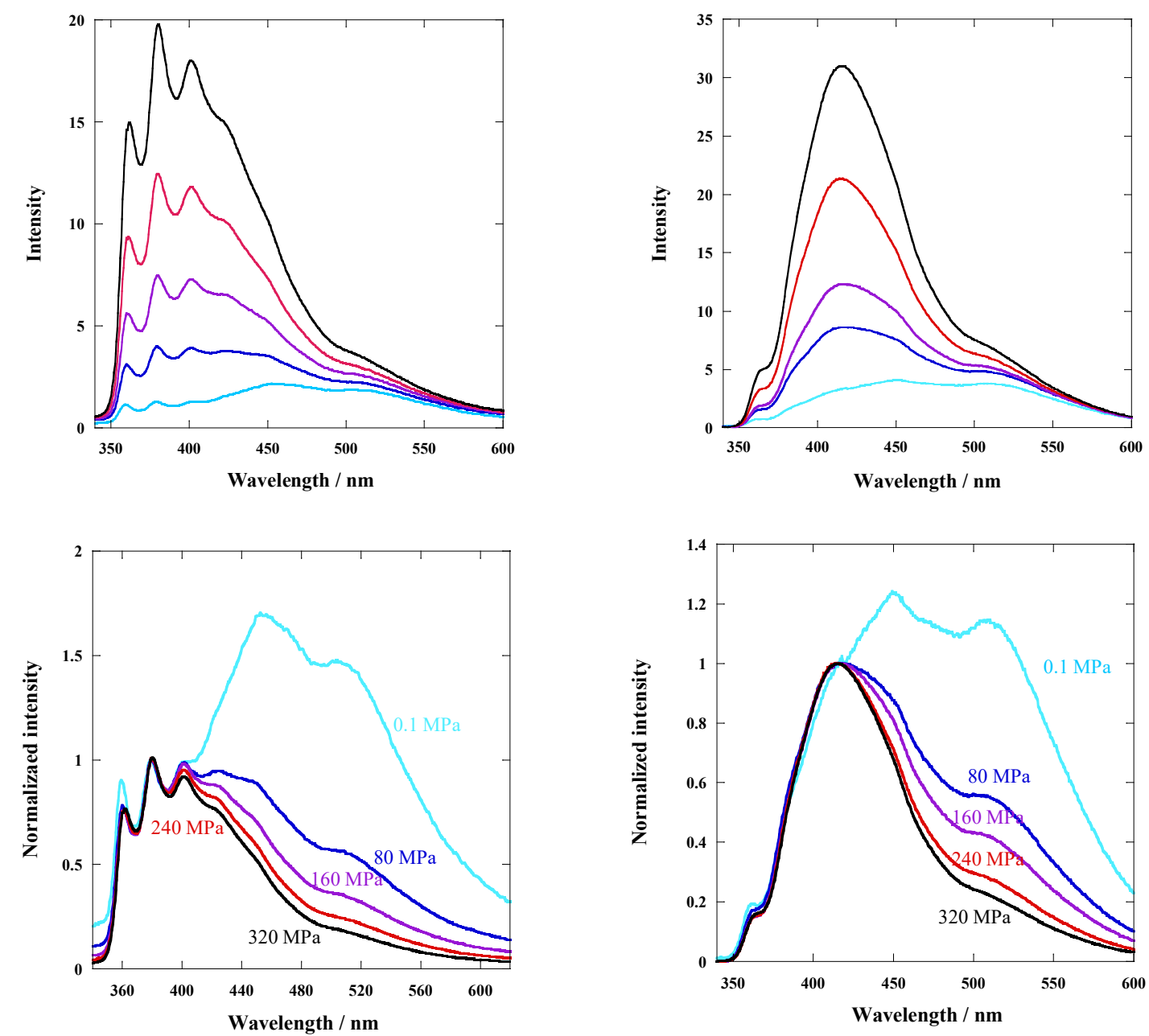

Figure 5 Changes in the fluorescence spectra of (A) TPE1 and (B) TPE2 measured in the aqueous phase. (A): $[\mathrm{TPE} 1]=500 \mu \mathrm{M}$ and $[\mathrm{CD}]_{\mathrm{w}}=300 \mu \mathrm{M}$. (B): [TPE2 $]=50 \mu \mathrm{M}$ and $[C D]_{\mathrm{w}}=500 \mu \mathrm{M}$. Normalized spectra of TPE species (TPE1; C and TPE2; D) in the twophase system. Applied pressure: 0.1, 80, 160, 240, and $320 \mathrm{MPa}$ (blue to black). 
A

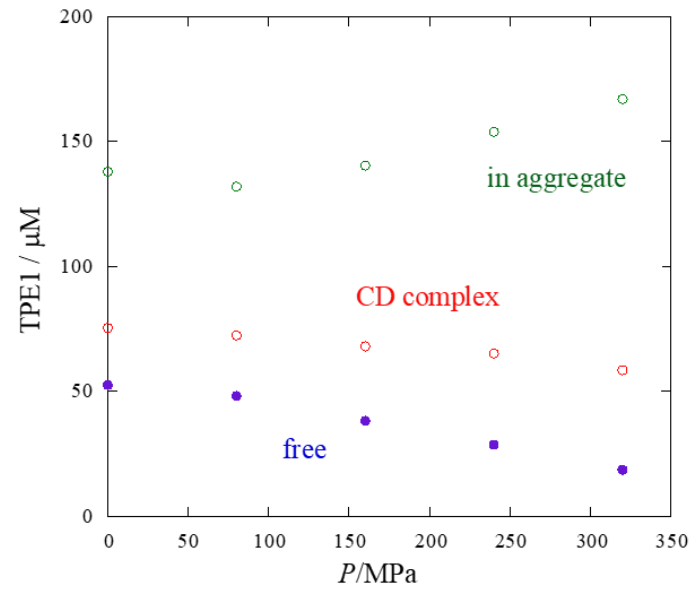

B

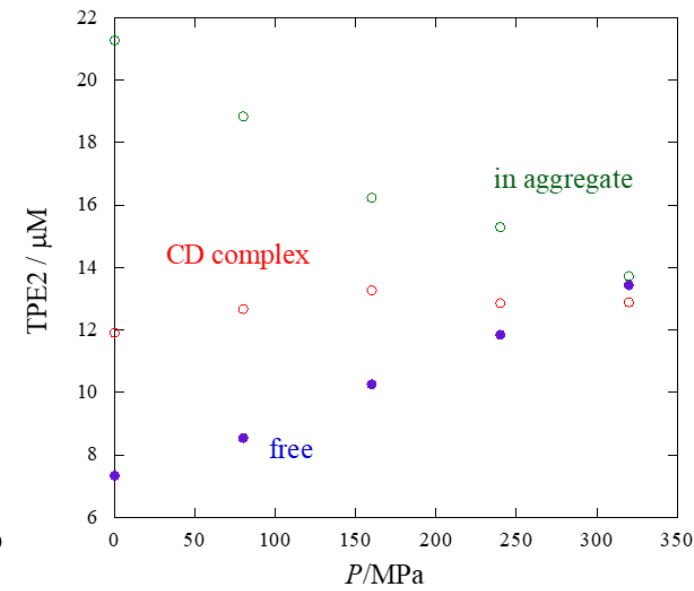

Figure 6 Concentrations of TPE species (TPE1; A and TPE2; B) in the aqueous phases. 
TOC Grafic

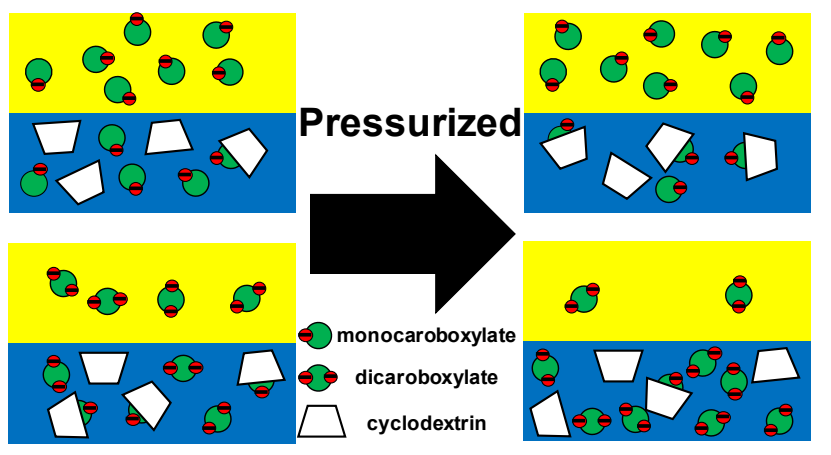




\section{Reference}

1. Flett, D. S., Solvent extraction in hydrometallurgy: the role of organophosphorus extractants. J. Organomet. Chem. 2005, 690, 2426-2438.

2. Xie, F.; Zhang, T. A.; Dreisinger, D.; Doyle, F., A critical review on solvent extraction of rare earths from aqueous solutions. Miner. Eng. 2014, 56, 10-28.

3. Tang, S.; Zhang, H.; Lee, H. K., Advances in Sample Extraction. Anal. Chem. 2016, $88,228-249$.

4. Sun, H.; Ge, X.; Lv, Y.; Wang, A., Application of accelerated solvent extraction in the analysis of organic contaminants, bioactive and nutritional compounds in food and feed. J. Chromatogr. A 2012, 1237, 1-23.

5. Asenjo, J. A.; Andrews, B. A., Aqueous two-phase systems for protein separation: a perspective. J. Chromatogr. A 2011, 1218, 8826-8835.

6. Hennion, M.-C., Solid-phase extraction method: development, sorbents, and coupling with liquid chromatography. J. Chromatogr. A 1999, 24, 3-54.

7. Poole, C. F., New trends in solid-phase extraction. TrAC, Trends Anal. Chem. 2003, 22, 362-373.

8. Bojczuk, M.; Żyżelewicz, D.; Hodurek, P., Centrifugal partition chromatography - A review of recent applications and some classic references. J. Sep. Sci. 2017, 40, 1597-1609.

9. Morley, R.; Minceva, M., Operating mode and parameter selection in liquidliquid chromatography. J. Chromatogr. A 2020, 1617, 460479.

10. Liang, C.; Lian, H.-Z., Recent advances in lipophilicity measurement by reversed-phase high-performance liquid chromatography. TrAC, Trends Anal. Chem. 2015, 68, 28-36.

11. Huang, X.-Y.; Di, D.-L., Chiral separation by counter-current chromatography. TrAC, Trends Anal. Chem. 2015, 67, 128-133.

12. Laforge, F. O.; Sun, P.; Mirkin, M. V., Shuttling Mechanism of Ion Transfer at the Interface between Two Immiscible Liquids. J. Am. Chem. Soc. 2006, 128, 1501915029.

13. Weatherly, C. K. T.; Ren, H.; Edwards, M. A.; Wang, L.; White, H. S., Coupled Electron- and Phase-Transfer Reactions at a Three-Phase Interface. J. Am. Chem. Soc. 2019, 141, 18091-18098.

14. Koynov, A.; Khinast, J. G.; Tryggvason, G., Mass Transfer and Chemical Reactions in Bubble Swarms with Dynamic Interfaces. AIChE J. 2005, 51, 2786-2800.

15. Gouaux, E.; MacKinnon, R., Principles of Selective Ion Transport in Channels and Pumps. Science 2005, 310, 1461-1465. 
16. Wilson, M. A.; Pohorille, A., Mechanism of Unassisted Ion Transport across Membrane Bilayers. J. Am. Chem. Soc. 1996, 118, 6580-6587.

17. Kenjereš, S., On Recent Progress in Modelling and Simulations of Multi-scale Transfer of Mass, Momentum and Particles in Bio-medical Applications. Flow, Turbul. Combust. 2016, 96, 837-860.

18. Abraham, J. P.; Gorman, J. M.; Sparrow, E. M.; Stark, J. R.; Kohler, R. E., A mass transfer model of temporal drug deposition in artery walls. Int. J. Heat Mass Transfer 2013, 58, 632-638.

19. Balakrishnan, B.; Tzafriri, A. R.; Seifert, P.; Groothuis, A.; Rogers, C.; Edelman, E. R., Strut Position, Blood Flow, and Drug Deposition: Implications for Single and Overlapping Drug-Eluting Stents. Circulation 2005, 111, 2958-2965.

20. Garg, S.; Rixey, W. G., The dissolution of benzene, toluene, m-xylene and naphthalene from a residually trapped non-aqueous phase liquid under mass transfer limited conditions. J. Contam. Hydrol. 1999, 36, 313-331.

21. Zhang, C.; Yoon, H.; Werth, C. J.; Valocchi, A. J.; Basu, N. B.; Jawitz, J. W., Evaluation of simplified mass transfer models to simulate the impacts of source zone architecture on nonaqueous phase liquid dissolution in heterogeneous porous media. $J$. Contam. Hydrol. 2008, 102, 49-60.

22. Poole, S. K.; Poole, C. F., Separation methods for estimating octanol-water partition coefficients. J. Chromatogr. B 2003, 797, 3-19.

23. Noble, A., Partition coefficients (n-octanol-water) for pesticides. $J$. Chromatogr. A 1993, 642, 3-14.

24. Berthod, A.; Carda-Broch, S., Determination of liquid-liquid partition coefficients by separation methods. J. Chromatogr. A 2004, 1037, 3-14.

25. Reymond, F.; Chopineaux-Courtois, V.; Steyaert, G.; Bouchard, G.; Carrupt, P.A.; Testa, B.; Girault, H. H., Ionic partition diagrams of ionisable drugs; $\mathrm{pH}$-lipophilicity profiles, transfer mechanisms and charge effects on solvation. J. Electroanal. Chem. 1999, 462, 235-250.

26. Floriano, W. B.; Nascimento, M. A. C., Dielectric Constant and Density of Water as a Function of Pressure at Constant Temperature. Braz. J. Phys. 2004, 34, 38-41.

27. Skinner, J. F.; Clussler, E. L.; Fuoss, R. M., Pressure Dependence of Dielectric Constant and Density of Liquids. J. Phys. Chem. 1968, 72, 1057-1064.

28. Kiselev, V. D.; Kornilov, D. A.; Konovalov, A. I., Changes in Permittivity and Density of Molecular Liquids under High Pressure. J. Phys. Chem. B 2014, 118, 37023709.

29. Matyushov, D. V.; Richert, R., Communication: Temperature derivative of the 
dielectric constant gives access to multipoint correlations in polar liquids. J. Chem. Phys. 2016, 144, 041102.

30. Schornack, L. G.; Eckert, C. A., The Effect of Pressure on the Density and Dielectric. J. Phys. Chem. 1970, 74, 3014-3020.

31. Samara, G. A., Temperature and Pressure Dependence of the Dielectric Constants of the Thallous Halides. Phys. Rev. 1968, 165, 959-969.

32. Takeda, Y.; Mizuno, H.; Okada, Y.; Okazaki, M.; Minakata, S.; Penfold, T.; Fukuhara, G., Hydrostatic Pressure - Controlled Ratiometric Luminescence Responses of a Dibenzo[a,j]phenazine - Cored Mechanoluminophore. ChemPhotoChem 2019, 3, 1203-1211.

33. Hegar, K.; Uematsu, M.; Franck, E. U., The Static Dielectric Constant of Water at High Pressures and Temperatures to $500 \mathrm{MPa}$ and $550^{\circ} \mathrm{C}$. Ber. Bunsenges. Phys. Chem. 1980, 84, 758-762.

34. Hara, K.; Kuwabara, H.; Kajimoto, O., Pressure Effect on Solvation Dynamics in Micellar Environment. J. Phys. Chem. A 2001, 105, 7174-7179.

35. Imoto, S.; Forbert, H.; Marx, D., Water structure and solvation of osmolytes at high hydrostatic pressure: pure water and TMAO solutions at 10 kbar versus 1 bar. Phys. Chem. Chem. Phys. 2015, 17, 24224-24237.

36. Williams, R. K., Pressure Enhancement of Charge-Transfer Complexing of 1Methyl-3-(carbomethoxy)pyridinium Cation and 8-Chlorotheophyllinate Anion in Aqueous Solution. J. Phys. Chem. 1981, 85, 1795-1799.

37. Isaacs, N. S.; Nichols, P. J.; Raston, C. L.; Sandova, C. A.; Young, D. J., Solution volume studies of a deep cavity inclusion complex of C60: [p-benzylcalix[5]arene $\subset$ C60]. Chem. Commun. 1997, 1839-1840.

38. Abou-Hamdan, A.; Bugnon, P.; Saudan, C.; Lye, P. G.; Merbach, A. E., HighPressure Studies as a Novel Approach in Determining Inclusion Mechanisms: Thermodynamics and Kinetics of the Host-Guest Interactions for $\alpha$-Cyclodextrin Complexes. J. Am. Chem. Soc. 2000, 122, 592-602.

39. Letcher, T. M.; Mercer-Chalmers, J. D.; Kay, R. L., Volume changes in complex formation between crown ethers of cryptand-222 and alkali metals in various solvents. Pure Appl. Chem. 1994, 66, 419-427.

40. Sueishi, Y.; Ide, T., A Characteristic Effect of Pressure on the Inclusion Complexation of Modified $\beta$-Cyclodextrins with 4-Substituted Phenols. Z. Phys. Chem. 2005, 219, 489-500.

41. Hong, Y.; Lam, J. W. Y.; Tang, B. Z., Aggregation-induced emission. Chem. Soc. Rev. 2011, 40, 5361-5388. 
42. Mei, J.; Hong, Y.; Lam, J. W.; Qin, A.; Tang, Y.; Tang, B. Z., Aggregationinduced emission: the whole is more brilliant than the parts. Adv. Mater. 2014, 26, 54295479.

43. Mei, J.; Leung, N. L.; Kwok, R. T.; Lam, J. W.; Tang, B. Z., Aggregation-Induced Emission: Together We Shine, United We Soar! Chem. Rev. 2015, 115, 11718-11940.

44. Song, S.; Zheng, H. F.; Li, D. M.; Wang, J. H.; Feng, H. T.; Zhu, Z. H.; Chen, Y. C.; Zheng, Y. S., Monomer emission and aggregate emission of TPE derivatives in the presence of gamma-cyclodextrin. Org. Lett. 2014, 16, 2170-2173.

45. Baglan, M.; Atilgan, S., Selective and sensitive turn-on fluorescent sensing of arsenite based on cysteine fused tetraphenylethene with AIE characteristics in aqueous media. Chem. Commun. 2013, 49, 5325-5327.

46. Nakamura, M.; Sanji, T.; Tanaka, M., Fluorometric Sensing of Biogenic Amines with Aggregation-Induced Emission-Active Tetraphenylethenes. Chemistry 2011, 17, 5344-5349.

47. Stewart, J. J. P., Optimization of parameters for semiempirical methods VI: more modifications to the NDDO approximations and re-optimization of parameters. J. Mol. Model 2013, 19, 1-32.

48. McDaniel, D. H.; Brown, H. C., An Extended Table of Hammett Substituent Constants Based on the Ionization of Substituted Benzoic Acids. J. Org. Chem. 1958, 23 , 420-427.

49. Kole, G. K.; Tan, G. K.; Vittal, J. J., Crystal engineering studies on the salts of trans-4,4'-stilbenedicarboxylic acid in the context of solid state $[2+2]$ cycloaddition reaction. CrystEngComm 2011, 13, 3138-3145.

50. Shahidi, F., Partial Molar Volumes of Organic Compounds in Water. Part 8.Benzene Derivatives. J. Chem. Soc., Faraday Trans. 1 1981, 77, 1511-1514.

51. Strong, L. E.; Bowe, M.; White, J.; Abi-Selah, K., Volumetric Properties of Some Methyl Substituted Benzoic Acids and Their Sodium Salts in Water as a Function of Temperature. J. Solution Chem. 1994, 23, 541-560.

52. Hoiland, H., Volumes of ionization of dicarboxylic acids in aqueous solution from density measurements at $25^{\circ}$ C. J. Chem. Soc. Faraday Trans. 1 1975, 71, 797-802. 53. Fasman, G. D., Eds; Circular Dichroism and the Conformational Analysis of Biomolecules; Springer, New York, 1996.

54. Fukuhara, G.; Mori, T.; Wada, T.; Inoue, Y., Entropy-Controlled Supramolecular Photochirogenesis: Enantiodifferentiating Z-E Photoisomerization of Cyclooctene Included and Sensitized by Permethylated 6-O-Modified $\beta$-Cyclodextrins. J. Org. Chem. 2006, 71, 8233-8243. 
55. Harata, K.; Uedaira, H., The Circular Dichroism Spectra of the $\beta$-Cyclodextrin Complex with Naphthalene Derivatives. Bull. Chem. Soc. Jpn. 1975, 48, 375-378.

56. Kodaka, M., A General Rule for Circular Dichroism Induced by a Chiral Macrocycle. J. Am. Chem. Soc. 1993, 115, 3702-3705.

57. Aree, T.; Chaichit, N., Crystal structure of $\beta$-cyclodextrin-benzoic acid inclusion complex. Carbohydr. Res. 2003, 338, 439-446.

58. Wei, Z.; Weiwei, Z.; Lihua, Z., Molecular simulation of ursolic acid $\gamma$ cyclodextrin host-guest inclusion compounds. BTAIJ 2014, 10, 1913-1916.

59. Millero, F. J., The effect of pressure on the solubility of minerals in water and seawater. Geochim. Cosmochim. Acta 1982, 46, 11-22. 